\title{
El examen vestibular abreviado, descripción, interpretación y análisis
}

\author{
The abbreviated vestibular exam, description, interpretation and analysis
}

\author{
Alejandro Peña M${ }^{1}$.
}

\section{RESUMEN}

Introducción: En el examen vestibular de un paciente vertiginoso juegan un rol muy importante las pruebas calóricas, pero la realización de éstas, unida a las nuevas pruebas que ha traído el avance tecnológico y computacional de la medicina, ha significado que el examen vestibular se ha convertido en la práctica diaria en un estudio muy largo y oneroso, por tal motivo parece útil la realización de un examen vestibular abreviado preliminar hecho en la misma consulta del médico que permita dar una orientación diagnóstica de bases sólidas.

Objetivos: El propósito de este estudio es la descripción, interpretación y análisis crítico del llamado "examen vestibular de 10 minutos del paciente mareado" y que permita llegar a conclusiones sobre la sensibilidad y especificidad de este examen abreviado.

Material y método: En este estudio, se describen, interpretan y analizan, según la información bibliográfica disponible, particularmente aquellos estudios de la medicina basada en evidencias las siguientes pruebas propuestas en el examen vestibular abreviado: Búsqueda del nistagmus espontáneo, búsqueda del nistagmus de mirada excéntrica, estudio de los movimientos oculares lentos o de rastreo ocular, estudio de los movimientos oculares rápidos o sacadas, test de Halmagyi, test de agitación cefálica, test de agudeza visual dinámica, test de inhibición de la fijación del nistagmus, maniobra de Dix y Hallpike, test de otoscopía neumática, estudio cerebeloso, estudio de la sensibilidad profunda, prueba de Romberg y estudio de la marcha. Debido a que varias de estas pruebas se efectúan en la prueba vestibular clásica sólo se analizan y discuten aquellas que no se efectúan de rutina en este examen.

Resultados: Las pruebas analizadas del examen vestibular abreviado revelan en su gran mayoría baja sensibilidad, menor de 50\%, pero una buena especificidad. Con algún entrenamiento básico son fáciles de realizar y requieren poco tiempo para su ejecución pero sí, mucho más que 10 minutos.

Discusión: Considerando el largo tiempo que requiere el especialista para efectuar un examen vestibular completo, un estudio básico resulta necesario para orientar la investigación clínica de un paciente vertiginoso. Las pruebas propuestas en este examen, tienen baja sensibilidad consideradas individualmente, pero la realización de todas ellas en su conjunto, le confiere al estudio una gran fortaleza y credibilidad, esto necesariamente unido a una muy buena anamnesis con cuestionario estructurado preestablecido.

${ }^{1}$ Facultad de Medicina, Universidad Católica del Maule. Talca, Chile. 
Conclusiones: El examen vestibular abreviado es un buen estudio, en que si bien las diferentes pruebas tienen baja sensibilidad, el resultado de la suma de todas ellas le confiere al examen una buena confiabilidad. Para su realización es indispensable un buen manejo de las pruebas y un buen conocimiento de la otoneurología por parte del examinador para su correcta interpretación. No existen estudios de medicina basada en evidencias de nivel 1 ó 2 que le otorguen alta credibilidad a estas pruebas. Este examen no debe reemplazar al examen vestibular clásico o formal, incluyendo las pruebas calóricas bi-termales, sino que deben ser consideradas como un estudio básico que permite orientar al especialista para tomar decisiones apropiadas diagnósticas y terapéuticas.

Palabras clave: Examen vestibular abreviado, examen vestibular básico, screening vestibular.

\section{ABSTRACT}

Introduction: Caloric tests play a very important role in a patient's vestibular exam. But these exams, along with the new test brought about by both technology and computing in Medicine, have turned these examinations into very long and expensive procedures. Taking all this into account, it seems suitable the realization of a preliminary vestibular exam performed in the doctor's consultation office. This will allow a diagnostic guideline with solid foundations.

Aim: To describe, interpret, and carry out a critical analysis of the so called "10 minute vestibular examination of the dizzy patient" in order to get to some conclusions about sensitivity and specificity of this short exam.

Material and method: Using the available bibliography, particularly medical studies based on evidence, the following test are described, interpreted and analyzed: spontaneous nystagmus, gaze nystagmus, smooth pursuit, saccades, Halmagyi test, head shake test, , dynamic visual acuity test, fixation suppression test, Dix-Hallpike maneuver, aural pressure/sound test, cerebellar limbs, somato-sensation test, Romberg test, and gait test. Since most of these tests are performed in the classic vestibular examination, only non-routine ones are analyzed and discussed.

Results: Most analyzed test show low sensitivity, under 50\%, but good specificity. With some basic training they are easy to carry out, and they require a short length of time, but over 10 minutes.

Discussion: Taking into consideration the long time the specialist needs for a vestibular examination, a basic vestibular study is highly necessary to guide the dizzy patient's clinical investigation. The tests suggested in this examination have a low sensitivity individually considered. But carrying out all of these together, added to a solid anamnesis with a preestablished structured questionnaire, gives the study strength and reliability.

Conclusion: The abbreviated vestibular examination is a good study. Although the various tests have a low sensitivity, the result of the addition of all of them, give the exam high reliability. To carry it out successfully, is essential to have a proper management of the test and a solid knowledge of neurotology, so that the examiner can make a good interpretation. No studies of Medicine based on evidence level 1 or 2 to give reliability to these test are available. This examination does not replace the classical examination, including bi-thermal caloric testing. It can be considered as a basic study aimed at guiding specialists to take suitable measures.

Key words: Abbreviated vestibular exam, Screening vestibular exam. 


\section{INTRODUCCIÓN}

En el estudio de un síndrome vertiginoso, ocupan un lugar importantísimo la anamnesis y el examen otoneurológico, y dentro de este último, posiblemente las pruebas calóricas tienen aun un rol fundamental. Estas pruebas calóricas sean hechas clásicamente con agua, o con aire con 0 sin electronistagmografía (ENG) 0 con 0 sin video-oculografía demandan al examinador un largo tiempo para su correcta ejecución e interpretación, lo que muchas veces restringe la capacidad de atención del especialista y más aún teniendo presente la creciente frecuencia de consultas de pacientes aquejados de vértigos que está, entre otros factores, en relación directa con el envejecimiento de la población en Chile.

En tal sentido parece atractiva la alternativa de poder realizar en estos pacientes primero un examen vestibular abreviado, básico en el mismo consultorio, que permita dar una orientación diagnóstica para después, dirigir y priorizar el estudio vestibular completo de estos enfermos, seleccionando así aquellos pacientes de mayor gravedad que requieran un estudio de laboratorio más profundo. Rutka el año $1998^{1}$ propone la aplicación de un examen vestibular simplificado que puede efectuarse en la consulta 0 al lado del lecho del paciente, el cual sería de gran ayuda diagnóstica. Goebel el año 2001, en su libro Practical management of the dizzy patient, sugiere la aplicación de una alternativa diagnóstica similar a la anterior pero ampliada que tituló, "The 10minute examination of the dizzy patient", o el examen de 10 minutos del paciente con mareos, lo cual encontró amplia acogida y popularidad particularmente en EE.UU. e Inglaterra idea que fue mejorada y corregida en una segunda edición de su libro el año $2008^{2}$ y que ha sido presentado por el autor como curso, en varias ocasiones en la Academia Americana de Otorrinolaringología y Cirugía de Cabeza y Cuello.

\section{OBJETIVOS}

El propósito de este estudio es, primero hacer una descripción de las pruebas vestibulares propuestas en este examen abreviado, segundo, describir la interpretación que se le puede dar a los resultados de estas pruebas y finalmente realizar un análisis crítico de las pruebas incluidas en este examen abreviado, enfatizando particularmente su sensibilidad, y su especificidad, según la mejor información disponible particularmente en estudios de la medicina basada en evidencias ${ }^{3}$ especialmente en Medline y Cochrane Data base, como fuentes de información.

\section{MATERIAL Y MÉTODO}

Para cumplir estos objetivos se procede primero a la descripción de las pruebas vestibulares propuestas por Goebel, luego a la interpretación de los resultados obtenibles de ellos, para después efectuar un análisis crítico de estos exámenes según la mejor información bibliográfica publicada, disponible.

\section{El examen vestibular abreviado}

En el examen de screening vestibular, o el examen de 10 minutos del paciente mareado, Goebel propone la utilización de 14 pruebas, que se pueden efectuar en la consulta médica o en el dormitorio del paciente. De éstas, varias de ellas, habitualmente se efectúan 0 se incluyen en el examen otoneurológico clásico o formal, razón por la cual dichos exámenes, no serán motivo de análisis 0 discusión. Curiosamente varias de estas otras pruebas que serán analizadas, habían sido descritas hace ya mucho tiempo, pero, o bien o no se incluyen de rutina en el examen habitual por una posible baja sensibilidad 0 especificidad de ellas $s^{4-7}$ 0 bien, si se les realiza, se les atribuye un bajo valor predictivo diagnóstico. Las pruebas propuestas por Goebel son las siguientes:

1. Búsqueda del nistagmus espontáneo (spontaneous nystagmus).

2. Búsqueda del nistagmus evocado de mirada excéntrica (gaze nistagmus).

3. Estudio de los movimientos oculares de seguimiento lento 0 de rastreo ocular (smooth pursuit).

4. Estudio de los movimientos oculares de seguimiento rápido o de sacadas (saccades).

5. Movimiento cefálico de empuje rápido, 0 de impulso cefálico (head thrust test). 
6. Test de agitación o sacudimiento cefálico (head shake test).

7. Test de agudeza visual dinámica, o búsqueda de oscilopsias (dynamic visual acuity test).

8. Test de inhibición visual del reflejo vestíbuloocular (fixation supresion test).

9. Maniobra de Dix y Hallpike (position test).

10. Estudio funcional de hemisferios cerebelosos 0 de neocerebelo (cerebellar cortical).

11. Prueba de Romberg (posture test).

12. Examen de la marcha (gait test).

13. Test de presión aural y vibración mastoídea (aural pressure/sound test).

14. Estudio de la propiocepción o de la sensibilidad profunda (somatosensation test).

\section{2) Búsqueda del nistagmus evocado de mirada excéntrica}

En esta prueba $^{2}$, se le solicita al paciente que mantenga su mirada sobre un objeto 0 blanco colocado a $20^{\circ}$ ó $30^{\circ}$ de la mirada central tanto a derecha como a izquierda durante 20 segundos, observándose el nistagmus originado por mirada excéntrica, cambios en su dirección, forma e intensidad del eventual nistagmus espontáneo.

Interpretación: La capacidad de mantener la mirada excéntrica está bajo el control del tronco cerebral y del vermis cerebeloso (paleocerebelo), particularmente el vestíbulo-cerebelo, y especialmente los lóbulos floculonodulares. Cuando estos mecanismos fallan en mantener la mirada excéntrica, el ojo tiende a irse a la línea media, seguido por sacadas de refijación sobre el objeto. Este nistagmus evocado de mirada excéntrica es de origen central y siempre bate hacia el objeto en observación. En cambio en el nistagmus espontáneo periférico (grado 3 de Alexander) la dirección de la fase rápida será siempre la misma. El nistagmus evocado de fijación excéntrica si bien tiene alta sensibilidad tiene el inconveniente de mostrar baja especificidad pues pueden registrarse con frecuencia falsos positivos teniendo como causas, la edad avanzada, uso de tranquilizantes, anticonvulsivantes, drogas y particularmente el alcohol.

Análisis: El nistagmus evocado de fijación excéntrica, no debe ser confundido con el nistagmus fisiológico de mirada lateral extrema (nistagmus de posición terminal 0 end-point nystagmus) que habitualmente se origina al mantener la mirada a $30^{\circ}$ a $60^{\circ}$, por más de 20 ó 30 segundos, lo cual constituye una limitante de esta prueba y que puede llevar a una confusión en su interpretación. También este nistagmus evocado puede manifestarse además de las lesiones del vermis cerebeloso, en tumores de tronco cerebral, lesiones degenerativas del SNC y en enfermedades neuromusculares como por ejemplo, la miastenia gravis.

Como dato anecdótico esta prueba es muy usada en EE.UU. por la policía para pesquisar conductores que manejan bajo el efecto del alcohol. Al respecto la legislación de algunos estados señala que la pesquisa de un nistagmus de fijación excéntrica en un conductor, permite deducir que existe $77 \%$ de posibilidades que el examinado tenga una alcoholemia superior al límite permitido para conducir un vehículo motorizado

\section{3) Estudio de los movimientos oculares de seguimiento lento o de rastreo ocular}

El estudio de los movimientos oculares lentos o de rastreo ocular está descrito hace ya mucho tiempo en el examen otoneurológico clásico, y para ello se usaba un péndulo que el paciente seguía con su mirada.

Este test se puede simplificar pidiéndole al paciente que siga con su mirada el movimiento lento del dedo del examinador a más o menos 90 $\mathrm{cm}$ de los ojos del paciente y teniendo si cuidado que el desplazamiento del dedo, no exceda los $60^{\circ}$ en sentido vertical y horizontal ${ }^{2}$.

Interpretación: En un sujeto normal el seguimiento ocular 0 de rastreo de un objeto en movimiento lento, genera un desplazamiento del globo ocular suave y sin sobresaltos. En las lesiones del tronco cerebral y cerebelo pueden observarse repetidas sacadas oculares en que el ojo parece perder el objeto en seguimiento y lo corrige con pequeñas sacadas. Sin embargo en la mayoría de los casos esta alteración no tiene valor de localización neurológica de la lesión, aunque alteraciones del seguimiento ipsilateral se han asociado a tumores del lóbulo parietal.

Análisis: Esta prueba es de difícil interpretación, pues es muy común encontrar un seguimien- 
to 0 rastreo ocular irregular en pacientes de edad avanzada, tanto es así que algunos autores consideran que no es de utilidad en pacientes mayores de 60 años, o que tengan mala visión, o estén bajo los efectos de sedantes; igualmente el cansancio, 0 falta de atención puede dar falsos positivos, estimándose así como una prueba de baja sensibilidad y de baja especificidad, como test único ${ }^{9}$.

\section{4) Estudio de los movimientos oculares de seguimiento rápido o de sacadas}

En esta prueba se le pide al paciente que fije su visión en forma alternada y rápida en dos puntos, por ejemplo un lápiz y la nariz del examinador a una distancia de alrededor de $50 \mathrm{~cm}$, de tal manera que las sacadas oculares sean aproximadamente de $15^{\circ}$, pudiendo observarse tres variables: la velocidad, la latencia de las sacadas y además el movimiento conjugado de los ojos ${ }^{2}$.

Interpretación: Los movimientos oculares sacádicos, son movimientos de refijación ocular en que participan los lóbulos frontales (sacadas voluntarias), la formación reticular del tronco cerebral (sacadas voluntarias e involuntarias), y los núcleos óculo-motores ${ }^{10}$. Un aumento en la latencia 0 retardo de inicio del movimiento se puede observar en lesiones corticales y del tronco cerebral, una lentitud en la velocidad de las sacadas se puede observar en lesiones de tronco cerebral, y una inseguridad en las sacadas especialmente exageradas o de hipermetría (overshoots) se pueden observar en lesiones del vermis cerebeloso y núcleo fastigio del cerebelo. Finalmente, movimientos oculares disconjugados con lentitud en la abducción ocular e hipermetría en la abducción se asocian con patología del fascículo longitudinal medial, frecuentemente asociada con la esclerosis múltiple.

Análisis: Estas alteraciones en las sacadas, particularmente las sacadas inseguras 0 exageradas o hipermetrías (overshoot), son frecuentes de observar en síndromes vertiginosos de origen central por el otoneurólogo y en tal sentido es necesario descartar lesiones del vestíbulo-cerebelo, aunque debe recordarse que esta prueba tiene baja sensibilidad.

Tanto el estudio de los movimientos oculares de seguimiento lento o de rastreo, como el estudio de las sacadas, representan dos sistemas básicos en los que se fundamenta la respuesta refleja óculo-motora, de ahí la importancia de su exploración.

\section{5) Movimiento cefálico de empuje rápido o de impulso cefálico, o prueba de Halmagyi}

Esta prueba conocida hace mucho tiempo por los neurólogos como el test de ojos de muñeca (doll's eyes test) y usada en el estudio de pacientes comatosos fue reintroducida y popularizada por Halmagyi y Curthoys en $1988^{11}$ para la pesquisa de alteraciones funcionales severas unilaterales de los canales semicirculares².

Para realizar este test se le pide al paciente que fije su mirada en la frente 0 nariz del examinador con sus ojos en la línea media para después girar rápidamente en $30^{\circ}$ la cabeza del paciente a izquierda y derecha. En un paciente sano los ojos describirán de inmediato un movimiento rápido, regular y seguro para centrar su visión en la nariz 0 frente del examinador; pero al existir un déficit 0 parálisis o paresia severa vestibular unilateral, los ojos harán pequeñas sacadas para refijar la mirada. Se recomienda observar los movimientos del iris de los ojos del paciente para pesquisarlos, pues estas sacadas pueden ser muy pequeñas y pasar inadvertidas por el examinador ${ }^{12}$.

Interpretación: Al girar la cabeza hacia el lado sano hay casi un perfecto movimiento conjugado de los ojos al lado opuesto para mantener la mirada fija, no requiriendo movimientos compensatorios oculares. Cuando existe un defecto del reflejo vestíbulo-ocular por ejemplo una parálisis vestibular, los movimientos rápidos cefálicos se asocian con un movimiento conjugado ocular compensatorio incompleto requiriendo movimientos de refijación o sacadas para estabilizar la mirada. Esto explica porque algunos de estos pacientes refieren espontáneamente en su anamnesis que al girar bruscamente su cabeza se les nubla la vista. Estas sacadas de refijación pueden ser bilaterales en casos de ototoxicidad.

Análisis: Según algunos autores esta prueba sería superior al Romberg y a la prueba de la indicación de Barany para pesquisar una paresia 0 parálisis vestibular unilateral. Sin embargo estudios de correlación con las pruebas calóricas muestran su debilidad. Así el valor predictivo en 
una paresia vestibular o sensibilidad es sólo de $68 \%$ al compararla con la prueba calórica, y la especificidad es de $90 \%{ }^{13}$.

Un estudio del subcomité de la Academia Americana de Neurología ${ }^{14}$, concluye que si bien éste es un estudio de costo cero, y fácil de realizar puede no detectar pérdidas vestibulares bilaterales 0 paresias vestibulares leves otorgándole un nivel de confiabilidad de clase III, según los cánones de la medicina basada en evidencias.

Sin embargo este test es considerado en algunos centros de estudio otoneurológicos europeos como "el único examen que se puede efectuar en la cama del enfermo 0 en la consulta del médico que permite la identificación del lado en la hipofunción del sistema vestibular periférico"15. Al respecto investigadores suizos han determinado una sensibilidad de $69,9 \%$ y una especificidad de $67,05 \%$ de este test ${ }^{15}$.

\section{6) Test de agitación o sacudimiento cefálico}

El test de agitación cefálica 0 de giros rápidos cefálicos, fue descrito el año 1907 por Barany ${ }^{16}$ pero extrañamente quedó en el olvido o no se incluyó en el examen otoneurológico formal 0 clásico por su baja sensibilidad, y sólo se reincorpora regularmente como método de screening vestibular o cribaje desde 1987, después de los trabajos de Hain ${ }^{17}$.

En este examen estando el paciente sentado se le pide que cierre sus ojos, se le inclina en $30^{\circ}$ su cabeza para así horizontalizar los canales semicirculares externos, luego se coge la cabeza y se efectúan movimientos enérgicos en giros de más 0 menos $45^{\circ}$ a derecha e izquierda, por alrededor de 15 segundos completando alrededor de 30 ciclos para después detenerse bruscamente. Después se le solicita al paciente que abra sus ojos, recomendándose que previamente se coloquen lentes de Frenzel de +15 ó +20 dioptrías para prevenir la fijación ocular del paciente ${ }^{1,2}$. Se considera como un resultado positivo la aparición de al menos 50 más batidas nistágmicas en dirección del oído sano que pueden extenderse hasta por 20 segundos. La pesquisa de este nistagmus señalaría la existencia de un déficit vestibular unilateral o la de un nistagmus espontáneo latente según la segunda ley de Ewald. La aparición de un nistagmus pervertido por ejemplo, vertical apuntaría a una lesión vestibular central ${ }^{18}$.

Interpretación: Durante la agitación cefálica el laberinto envía información de actividad neural al tronco encefálico la cual "carga" los circuitos vestibulares centrales. Este fenómeno es llamado "velocidad de almacenamiento" en neurofisiología. Cuando la agitación cefálica se detiene, la actividad neural almacenada es descargada en un corto período de 5 a 20 segundos llamado "constante de tiempo del reflejo vestíbulo-ocular". Si las entradas de los dos laberintos vestibulares durante la agitación cefálica han sido simétricas, las descargas de los circuitos vestibulares se neutralizarán y no habrá nistagmus. Sin embargo si las entradas han sido asimétricas aparecerá un nistagmus².

Aquellos pacientes con lesiones severas unilaterales y reducción de su función, tendrán un nistagmus que puede durar de 5 a 20 segundos que corresponde al tiempo de descarga, en dirección del oído sano, y dependiendo de la intensidad del nistagmus inicial, podrán tener un segundo nistagmus más breve que batirá en sentido contrario, hacia el oído enfermo. En cambio en lesiones vestibulares agudas, el test será negativo por un bloqueo temporal del sistema de velocidad de almacenamiento.

Análisis: Este test ha sido motivo de muchas críticas por su baja sensibilidad, al respecto uno de los pocos estudios publicados en lengua española, señala que la sensibilidad del test para determinar la existencia de hiporreflexia vestibular es sólo de $48 \%$, pero con una buena especificidad de $95 \%$. Experiencia que se repite en otros estudios ${ }^{19}$.

Estos resultados llevaron al Subcomité de la Academia Americana de Neurología a considerarla como un método no cuantitativo, que no permite detectar lesiones vestibulares bilaterales, ni tampoco lesiones vestibulares leves 0 de mediana severidad, incluyéndola en la categoría III de la medicina basada en evidencias ${ }^{14}$.

\section{7) Test de agudeza visual dinámica u oscilopsias}

Pacientes con lesión o hipofunción vestibular bilateral, se quejan habitualmente de oscilopsias (no vértigos), especialmente al atardecer 0 en la, noche, durante la marcha, por ejemplo, en los casos de ototoxicidad o de enfermedad de Méniére bilate- 
ral. Para comprobar la normalidad del reflejo véstibulo-ocular se le mide primero, la visión al paciente en reposo en una cartilla de Snellen, para luego pedirle que lea nuevamente la cartilla mientras gira su cabeza en un arco de $60^{\circ}$ con una velocidad de 2 ciclos por segundos o 2 Hertz. Normalmente la pérdida de agudeza no debe bajar en más de tres líneas, pérdidas mayores de tres líneas se presentan en lesiones vestibulares bilaterales $^{1,2}$.

Interpretación: El test de agudeza visual dinámica es un buen examen para monitorear la función vestibular de aquellos pacientes que están recibiendo aminoglicósidos o quimioterápicos por ejemplo cisplatino, y que el médico tratante no lo sospecha al estar el paciente generalmente en reposo en cama durante su tratamiento ${ }^{20}$.

Análisis: El test de agudeza visual dinámica para el estudio de hipofunción vestibular bilateral ha demostrado al respecto, ser una buena prueba para pesquisar este déficit, Longridge refiere una sensibilidad de $89,7 \%$ al comparar los resultados con sujetos normales y una especificidad de $93.5 \%{ }^{21}$.

\section{8) Test de inhibición visual del reflejo vestíbulo-ocular}

El test de supresión visual del reflejo vestíbuloocular, permite conocer la capacidad del SNC de suprimir visualmente una señal vestibular. Si a un paciente se le pide que siga un objeto que rota simultáneamente con su cabeza en la misma dirección, los ojos deberán permanecer estables en sus órbitas debido a la fijación visual y a la supresión del reflejo vestíbulo-ocular. Al existir una patología central particularmente del vestíbulo-cerebelo, aparecerá un nistagmus por falla del mecanismo inhibitorio central, este fenómeno es similar a la falla de la inhibición del nistagmus en la prueba calórica con la fijación ocular en las patologías vestibulares centrales. Este examen se puede efectuar de dos maneras, la primera es pedirle al paciente que se encuentre sentado en una silla giratoria de escritorio, que extienda sus brazos con sus pulgares unidos y que fije su mirada en ellos y en ese momento girar la silla del paciente a $2 \mathrm{~Hz}$ hacia derecha e izquierda, y la segunda es pedirle al paciente que sostenga entre sus dientes una regla de $50 \mathrm{~cm}$ y solicitarle que fije su mirada en el extremo distal de ésta para después girar su cabeza en movimientos rápidos a izquierda y derecha, observando la eventual aparición de un nistagmus ${ }^{1,2,22}$.

Interpretación: La pesquisa de un nistagmus en este test obedece como se señaló al mismo fenómeno fisiopatológico de la aparición de un nistagmus con la fijación ocular en la prueba calórica, y siempre debe sospecharse una lesión del flóculus cerebeloso, o del SNC, mientras no se encuentre otro origen.

Análisis: Una de las características fundamentales en una persona normal es la capacidad de reducir la intensidad del nistagmus a través de la fijación ocular, la que se pierde en algunas patologías del SNC, sin embargo según Barín ${ }^{22}$ y otros investigadores, los test para su investigación tienen numerosas limitaciones que afectan su utilidad, con baja sensibilidad y baja especificidad y requieren una cuidadosa interpretación para evitar falsos positivos y falsos negativos. Al respecto se ha observado que incluso con dosis muy bajas de alcohol el examen puede dar resultados de falsos positivos ${ }^{24}$, así como también la ingesta de fármacos con efecto depresor del SNC.

\section{RESULTADOS Y DISCUSIÓN}

El gigantesco avance tecnológico y computacional de las últimas décadas en medicina, ha permitido incorporar al examen otoneurológico, nuevos test o pruebas tales como la video-oculografía, la posturografía dinámica computarizada, el VEMP, la prueba rotatoria sinusoidal de aceleración armóni$\mathrm{ca}$, la RM etc. Io que ha significado un enorme progreso para la pesquisa precoz y confiable de la patología vestibular.

Sin embargo los aspectos negativos de la incorporación de estos nuevos exámenes son varios, primero ha encarecido el examen otoneurológico, hecho que se repite en general en todas las ramas de la medicina, con el uso de nuevas tecnologías; segundo, la posibilidad de poder realizar nuevos exámenes para el estudio de la patología vestibular, conlleva a que éstos requieren muchísimo más tiempo para efectuarlos, lo que ha significado que la mayor parte de los otorrinolaringólogos solicita 
estos exámenes a tecnólogos médicos o bien porque no tiene tiempo para efectuarlos 0 porque carece de los equipos adecuados para su realización o porque considera a estos equipos indispensables para llegar a un diagnóstico correcto, llevando así a la deshumanización de la medicina y a la pérdida de la importante relación médico-paciente.

Lejanos están los tiempos del destacado otoneurólogo chileno, Santiago Riesco Mac Clure ${ }^{7}$, quien con escasísimos medios técnicos destacó en el medio científico mundial con sus brillantes aportes al conocimiento de la patología vestibular. En tal sentido la realización por parte del otorrinolaringólogo en su propia oficina de un examen vestibular abreviado permitiría rescatar o recuperar este estudio que debe ser siempre parte de la práctica médica diaria de todos los especialistas y no delegar toda esta investigación al tecnólogo médico.

De la revisión de todos estos exámenes discutidos y analizados, se puede observar que casi ninguno de ellos tiene una sensibilidad alta que se acerque al $100 \%$, pero no así la especificidad que muestra mejores niveles de confianza en casi todas estas pruebas, sin embargo con la realización del conjunto de todos estos exámenes propuestos por Goebel², es posible llegar a conclusiones con un alto grado de certeza lo que unido a una muy buena anamnesis, ojalá con un cuestionario preescrito para no olvidar información importante que oriente al diagnóstico, es posible dirigir el estudio del paciente y así poder pesquisar aquellas patologías que requieran un estudio de laboratorio o instrumental de mayor profundidad que llevará a un diagnóstico correcto y a un buen resultado terapéutico.

Otro aspecto importante de considerar en la realización del examen vestibular abreviado o de oficina es, que si bien éste es aparentemente simple, requiere por parte del especialista como elemento básico un buen conocimiento de la patología vestibular, y una acabada práctica clínica de estas pruebas para así poder lograr una correcta interpretación de los resultados que conducirán a una hipótesis diagnóstica correcta.

\section{CONCLUSIONES}

Del estudio y análisis de las pruebas propuestas en el examen vestibular abreviado, y de acuerdo con la información bibliográfica disponibles se puede concluir lo siguiente:

1. La mayor parte de las pruebas analizadas se caracterizan por tener un bajo perfil de sensibilidad lo que las hace al considerarlas individualmente poco confiables, salvo la prueba de Halmagyi, en que todos los estudios le confieren una alta sensibilidad.

2. Más de $50 \%$ de las pruebas analizadas demuestran una buena especificidad con bajos niveles de falsos positivos.

3. No se encontró ningún trabajo en la literatura revisada en Medline y Cochrane Data Base, de otorrinolaringología basada en evidencias de nivel 1 a (metaanálisis de estudios prospectivos controlados randomizados), ni de nivel $1 \mathrm{~b}$ (evidencia de al menos un estudio prospectivo randomizado controlado) ni tampoco de nivel 2. Todos los trabajos revisados no superan el nivel 3, es decir evidencias de estudios bien diseñados descriptivos no experimentales por ejemplo estudios de casos o comparativos, de manera que los niveles de confianza de estos estudios son medianos ${ }^{25}$.

4. Del análisis de estos estudios se desprende la importancia fundamental de una buena anamnesis ${ }^{26}$ al respecto estudios sobre la capacidad predictiva de la anamnesis han demostrado una capacidad predictiva del vértigo migrañoso de $92 \%$, del vértigo postural paroxístico benigno de $90 \%$, de la enfermedad de Méniére de $86 \%$, de la neuronitis vestibular de $63 \%$, otorgándole un valor predictivo general a la anamnesis de $84 \%^{27}$. De allí la importancia de un cuestionario estructurado en la evaluación de estos pacientes.

5. Es la suma de la información recogida de las 14 pruebas del examen vestibular abreviado, la adecuada interpretación de sus resultados y las conclusiones que de ellos se desprenden lo que otorga a este examen una alta confiabilidad diagnóstica y que unido a una correcta anamnesis, pueden permitir al otorrinolaringólogo tomar decisiones adecuadas en el manejo de un paciente aquejado de un síndrome vertiginoso.

6. Finalmente este examen vestibular abreviado no puede reemplazar al examen otoneurológico clásico ni menos a las pruebas calóricas bi- 
termales que juegan un rol muy importante en el diagnóstico de patologías vestibulares periféricas y centrales ${ }^{28}$, pero sí constituye este examen abreviado una herramienta diagnóstica muy útil para orientar la toma de decisiones adecuadas diagnósticas y terapéuticas.

\section{BIBLIOGRAFÍA}

1. RUTKA, John. Evaluation of Vertigo. En: Blittzer Andrew, Pillsbury Harold, Jahn Antony, Binder William. Office -baseded surgery in Otolaryngology. New York: Thieme Medical Publishers, 1998; 71-8.

2. Goebel Joel A. The 10-minute Examination of the dizzy patient En: Goebel Joel. Practical management of the dizzy patient. Second edition. Philadelphia: Lippincott Williams \& Willkins, 2008; 437-40.

3. Shin Jennifer J, Hartnick Christopher J, Randolph Gregory. Evidence-based otolaryngology. New York: Springer Science \& Business Media, 2008.

4. Cárdenas José Luis. Exploración Clínica Vestibular. En: Morales C. Otoneurología Clínica. Santiago: Editorial Universitaria, 1992; 93-107.

5. Roquette J, Sierra G, Bartual J. Semiología del vértigo. Exploración del paciente vertiginoso. En: Ramírez Camacho R. Trastornos del equilibrio. Un abordaje multidisciplinario. Madrid: Mc Graw-Hill. Interamericana de España SAU, 2003; 21-48.

6. Barona de Guzmán R, Martín Sanz e, Platero ZamarreÑo A. Exploración de la función vestibular. En: Suárez C, Gil-Carcedo LM, Marco J, Ortega P, Trinidad J. Tratado de Otorrinolaringología y Cirugía de Cabeza y Cuello. $2^{\mathrm{a}}$ edición. Madrid: Editorial Médica Panamericana SA, 2007; 1187-208.

7. Riesco Mac Clure JS, Velasco R. Otoneurología. En: Alonso JM, Tato JM. Tratado de Otorrinolaringología y Broncoesofagología. $3^{\mathrm{a}}$ edición. Madrid; Editorial Paz Montalvo, 1976; 495-528.

8. Horizontal Gaze Nystagmus: The Science \& the law. A resource guide for judges, prosecutors and law enforcement. Disponible en: http:// www.nhtsa.gov/people/injury/enforce/ nystagmus/. Consultado el 29/01/2011.
9. Paquette C, Fung J. Old age affects gaze and postural coordination. Gait Posture 2010; Dec 4.

10. Sparks David L. The brainstem control of saccadic eye movements. Neuroscience-Natural Rev 2002; 3: 952-64.

11. Halmagy GM, CuRThoys IS. A clinical sign of canal paresis. Arch Neurol 1988; 45: 737-9.

12. WuYTs F. Principle of the head impulse (thrust) test or Halmagyi head thrust test (HHTT). $B$ ENT 2008; 4.Supp.8: 23-5.

13. Brandt Thomas, Strupp Michael. General vestibular testing. Clinical Neurophisiol 2005; 116: 406-26.

14. Fife TD, Tusa RJ, Furman JM, Zee DS, Frohman E, ET AL. Assessment: Vestibular testing techniques in adults and children. Neurology 2000; 55: 1431-41.

15. Jorns-Häderly $M$, Straumann $D$, Palla $A$. Accuracy of the bedside head impulse test in detecting vestibular hypofunction. J Neurol Neurosurg Psychiatry 2007; 78: 1113-8.

16. BáRánY R, citado por Hain Timothy en: Head Shaking Nystagmus. Disponible en: www.dizziness-and-balance.com/research/hsn/ Head\%20Shaking\%20Nystagmus.htm. Last modified Nov 21, 2009. Consultado el 29/01/ 2011.

17. Hain TC, Fetter M, Zee DS. Head-shaking nystagmus in unilateral peripheral vestibular lesions. American J Otolaryngology 1987; 8: 36-47.

18. BoNIVER R. Head shaking nystagmus. B-ENT 2008; 4,Suppl 8: 9-12.

19. Pérez P, Rodríguez N, Sequeiros S, Llorente J, Gómez J, Suárez C. Utilidad del nistagmo de agitación cefálica en la exploración vestibular clínica básica. Acta Otorrinolaringol Esp 2005; 56: 300-4.

20. Haln TC. Dynamic visual acuity test. Disponible en: www.tchain.com./otoneurology/practice/ dynvisual.html. Consultado el 29/01/2011.

21. Longridge NS, Mallison Al. The dynamic illegible E test: a simple technique for assessing the ability of the vestibule-ocular reflex to overcome vestibular pathology. J Otolaryngol 1987; 16: 97-103.

22. Hain C Tімотнy. Testing of fixation suppression Disponible en: http://www.dizziness-and- 
balance.com/practice/fixation.html. Last modified June 18, 2009. Consultado el 29/01/2011.

23. Barin KamRan. The Fixation Suppression Test in ENG Evaluation. Disponible en: http://www.audiologyonline.com/articles/article_detail.asp?article_Consultado el 29/01/2011.

24. HaRder T, ReKer U. Influence of low dose alcohol on fixation suppression. Acta Oto-laryngolog 1995; 115 (s520): 33-6.

25. Hamid AM. Prediction of ENG results from
Office Vestibular Examination. OtolaryngolHead and Neck Surg 2006; 135: 2s.

26. Bonavita B, De Simone R. Le vertigini: dalla storia clínica alle ipotesi diagnostiche. Neurol Sci 2004; 25: S1-S6.

27. Zhao JG, Piccirillo Jf, Spitznagel EL, Goebel JK. Predictable capability of historical data for diagnosis of dizziness. Oto Neurotol 2011; 32(2): 284-90.

28. Corvera J. Neurotología clínica. $2^{\mathrm{a}}$ edición. México; Salvat editores. 1990. 